\title{
Guaranteed Ray Intersections with Implicit Surfaces
}

\author{
Devendra Kalra \\ Alan H. Barr \\ Computer Science Department \\ California Institute of Technology \\ Pasadena, California 91125
}

\begin{abstract}
In this paper, we present a robust and mathematically sound rayintersection algorithm for implicit surfaces. The algorithm is guaranteed to numerically find the nearest intersection of the surface with a ray, and is guaranteed not to miss fine features of the surface. It does not require fine tuning or human choice of inter active parameters. Instead, it requires two upper bounds: " $L$ " that limits the net rate of change of the implicit surface function $f(x, y, z)$ and " $G$ " that limits the rate of change of the gradient. We refer to an implicit surface with these rate limits as an "LG-implicit surface."

Existing schemes to intersect a ray with an implicit surface have typically been guaranteed to work only for a limited set of implicit. functions, such as quadric surfaces or polynomials, or else have been ad-hoc and have not been guaranteed to work. Our technique significantly extends the ability to intersect rays with implicit surfaces in a
\end{abstract} guaranteed fashion.

CR Categories and Subject Descriptors: I.3.3 (Picture/Image Generation) - display algorirthms; I.3.5 (Computational Geometry and Object Modeling) - Curve, surface, solid and object representations, Geometric algorithms, languages and systems; I.3.7 (ThreeDimensional Graphics and Realism) - Color, shading, shadowing and texture, Visible line / surface algorithms;

Keywords: Implicit Surfaces, Ray Tracing, Rendering, Sampling, Subdivision, Lipschitz Constant.

\section{Introduction}

The task of intersecting rays with implicit surfaces (see Figure 1) is an important part of the theory and application of ray casting and ray tracing. Implicit functions can be used to represent some useful and interesting surfaces. They also have a nice composition property in that they can be combined in geometrically useful and concise ways to generate new implicit functions. Examples of these are the algebraic surfaces introduced by Blinn [BLINN 82] and extensions (Figure 16 and 20).

The reader might be surprised to find that it is impossible to create an algorithm (based solely on the evaluation of the implicit function at points in space) which is guaranteed to correctly intersect a ray with an arbitrary implicit surface. For any algorithm that could be constructed, there exist troublesome functions $f(x, y, z)$ for which important parts of the surfaces are missed. Figure 2 illustrates this problem. In this example, we would need information about the existence of a spike in between the sample points, which is not available from the values of the function obtained at the sample points.

Thus, to guarantee ray intersections with implicit surfaces, some sort of auxiliary information is needed. In our case, we have chosen this auxiliary information to be the $L$ and $G$ rate limits.

Permission to copy without fee all or part of this material is granted provided that the copies are not made or distributed for direct commercial advantage, the ACM copyright notice and the title of the publication and its date appear, and notice is given that copying is by permission of the Association for Computing Machinery. To copy otherwise, or to republish, requires a fee and/or specific permission.

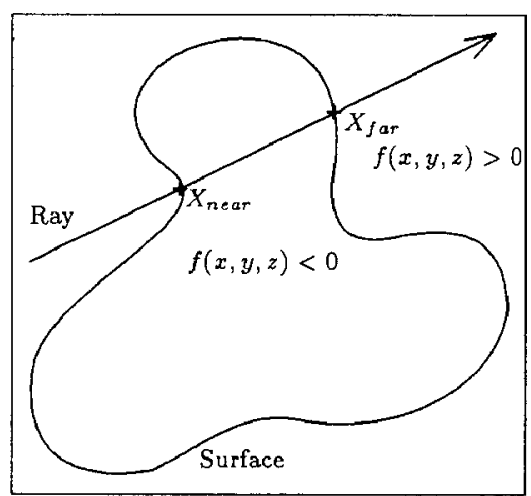

Figure 1: An implicit surface is defined by $f(x, y, z)=0 . f(x, y, z)$ is negative inside the surface, positjve outside the surface and zero on the surface. The ray intersects the surface at $X_{n e a r}$ and $X_{\rho a r}$.

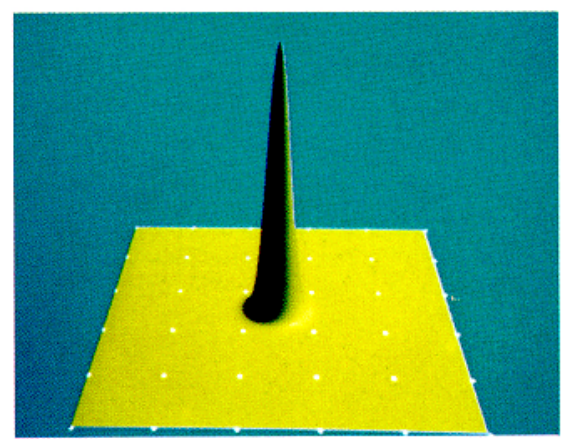

Figure 2: A spike function demonstrates some of the difficulties of sampling without sufficient information about the function being sampled. The above surface at each of the samples has zero curvature; the samples do not indicate the presence of a spike since the spike falls between the sampling points.

\subsection{A Need For "Guaranteed" Algorithms}

Some readers might question the utility of developing an apparently complex algorithm using the mathematical machinery of $L$ and $G$ bounds. They could also question the need to directly render complex modeling primitives, instead of converting into more easily renderable objects such as polygons. Finally, they might question the utility of implicit functions at all. They could believe that there would be no need for surface types beyond bicubic patches, quadric surfaces, and polygons for their modeling needs.

The answer to the first question is straightforward. Given the $L$ and $G$ numeric rate constants, our algorithm actually is quite simple and easy to implement. The amount of code for the algorithm is relatively small and is easily put into a standard ray tracing program.

More importantly, however, the algorithm guarantees that we will find all of the correct ray intersections in the entire image. If we were to first convert the implicit model into polygons, we would have to produce a "guaranteed" polygonal tesselation of the surface. This is 
a non-trivial task for general implicit surfaces, and could depend on the camera view angles and viewing parameters of the scene. (Our technique can be adapted to produce guaranteed polygonalizations, but we feel direct rendering is easier).

Even in directly rendering a geometric primitive, rather than its polygonal tesselation, a "guaranteed" technique, which does not require any corrective interaction or fine tuning of parameters by a human being, is most valuable. This is especially attractive to people making computer animation. In fact, we frequently use computer animation as a means to verify the accuracy of our rendering algorithms. Imagine running long scripts to make a movie and then finding that frame number 129 has pixel dropouts because the frame uses a particular camera angle and parameters for the rendering algorithm that worked for other frames but do not work for this frame. The recourse is to find the right set of parameters for this frame and restart the animation until we notice other anomalies. With a guaranteed "direct" method, one is assured of the correctness of the whole sequence, without re-rendering frames.

Even in making still-frames, much time is spent modeling a scene. With an algorithm that guarantees the correctness of the rendering there is less to worry about, as changes are made to other object parameters of the scene.

The utility of having a wider variety of surface types can be seen in Figures 16 and 20. The polygonal or patch representation for these objects would be more difficult to specify and would require more data than implicit techniques.

Finally, developing a "direct" guaranteed algorithm that works every time without any searches through a parameter space was an exhilarating research experience for us.

1.2 Previous Methods To Render Implicit Surfaces An implicit surface $S$ is defined by a function $S: f(\mathbf{x})=0$. The function is negative inside the surface and positive outside (Figure 1).

Polynomial Implicit Functions

The efforts to render implicit surfaces have been two-fold. The first approach has been to limit types of implicit functions. [HANRAHAN 83] limited the implicit functions to be polynomial functions of spatial variables. Using some results presented in [USPENSKY 48] and [COLLINS and AKRITAS] and a theorem due to Descartes, this method gurarantees the nearest intersection of a ray with a polynomially defined implicit surface. [MIDDLEDITCH et al 85] have also used polynomially defined surfaces to generate some kinds of blend surfaces.

Polygonizing Implicit Surfaces

The second approach has tried to render more general implicit surfaces by polygonizing implicit surfaces.

There is a basic difficulty with using a naive polygonization of a surface. With a view independent polygonization algorithm, we could either end up rendering too many polygons when the object covers very small number of pixels on the screen or rendering too few polygons when the objects covers a large area on the screen. [VON HERZEN 87] has attempted to deal with view dependent polygonization. On the other hand, ray casting automatically samples an object at a level that depends on the object's coverage of the screen.

It is also more natural and easier to model some objects in one modeling domain than another. The pictures in this paper have been modeled in a sculptor's paradigm of adding a little clay here or removing a little clay there. This can be done most naturally with blended implicit functions.

However, some researchers have polygonized impłicit surfaces with varying success. The schemes that try to polygonize implicit surfaces compute the value of $f(\mathbf{x})$ defining the surface for points $P_{\mathrm{i}}$ on a grid These methods consider segments at whose two endpoints $f(x)$ has opposite signs. Then the intersection of $S$ with this segment is approximated by various methods such as linear interpolation. These "intersections" along the edges of the rectangular boxes forming the sampling lattice are then connected according to some heuristics to form polygons. These polygons are then rendered. See [WYVILL 87[1]], [WYVILL 87[2]], and [BLOOMENTHAL 87].

Forming polygons out of approximate intersections along the box edges is heuristic and leaves ambiguities in many cases when more (a)
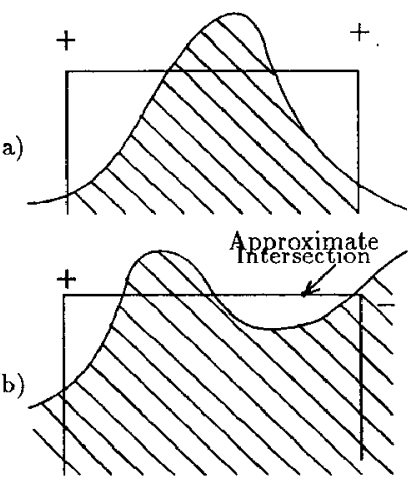

Figure 3: Possible problems with a naive Implicit Surface Polygonizing Algorithm; (a) small features are liable to missed because both sample points are outside the surface, (b) incorrect intersections may be computed because of strange behaviour of the function near the sample points.

than one possibility exists. A second more important problem with the above algorithm is that they are based only on the values of the implicit function at certain points. As noted before, this means that features finer than the sampling grid will be either completely ignored (Figure $3(\mathrm{a})$ ) or the approximate intersections formed will be grossly incorrect (Figure $3(\mathrm{~b})$ ). There is not enough information in the function values even to provide an estimate of the size of the sampling grid.

In section two, we describe our mathematical approach. In section 3 , we describe an overview of the algorithms, while in section 4 we describe the algorithms in more detail. In sections 5 and 6 , we describe our results and conclusions, and provide supplemental mathematical information in the appendices.

\section{Our Approach: Creating LG-Surfaces}

We present an algorithm that

a) Guarantees that the smallest features of the surface are sampled.

b) Obtains the nearest intersection of a ray from the origin of the ray with the implicit surface $S$ represented by the implicit function $f(\mathbf{x})$.

We do not generate polygons, but obtain guaranteed numerical intersections of rays with the implicit surface.

\subsection{Mathematical Preliminaries:}

We first present some mathematical definitions and preliminaries:

\section{Definition of Implicit Surface:}

A general implicit surface $S$ is defined by (See Figure 1)

$$
f(x, y, z)=0
$$

or, in vector notation,

$$
f(\mathbf{x})=0
$$

Ray Definition

We define a ray via:

$$
\mathbf{x}=\alpha t+\beta, \quad t \geq 0,
$$

where vector $\alpha$ is a unit vector ray direction and vector $\beta$ is the origin of the ray corrsponding to $t=0$.

Definition of $F(t)$

Given a function $f(\mathrm{x})=0$ of spatial variables $\mathrm{x}=(x, y, z)$, we substitute

$$
\mathrm{x}=\alpha t+\beta
$$

into $f(\mathbf{x})$ to define

$$
F(t)=f(\alpha t+\beta)
$$




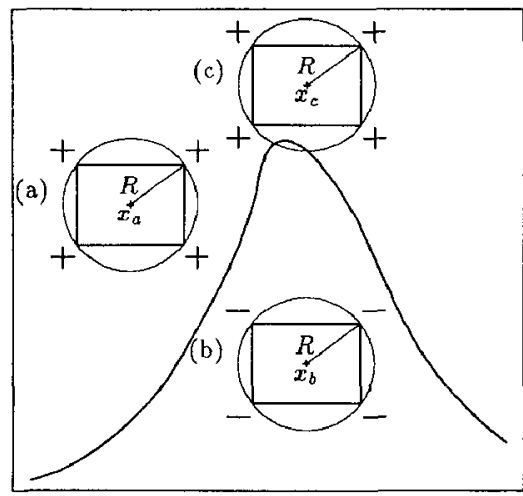

Figure 4: Given a point $x_{0}, r=f\left(x_{0}\right) / \mathcal{L}$ is the radius of the sphere $S$ around $x_{0}$ such that $f(x)$ does not change sign in $S$. $\mathcal{L}$ is the Lipschitz constant in equation 3 in the region of $S$. Spheres (a) and (b) are guaranteed not to intersect the surface since Lipschitz radii $r_{a}=f\left(x_{a}\right) / \mathcal{L}_{a}<R$ and $r_{b}=f\left(x_{b}\right) / \mathcal{L}_{b}<R$. However, $r_{c}>R$ and $S_{c}$ may intersect the surface.

\section{Definition of $g(t)$}

We now define a new function

$$
\begin{aligned}
g(t) & =\frac{d F}{d t} \\
& =\left.\boldsymbol{\alpha} \cdot \nabla f(\mathbf{x})\right|_{\alpha t+\beta}
\end{aligned}
$$

Note that $g(t)$ is the directional derivative of $f(x)$ along the ray direction $\alpha$ (' $g$ ' relating to "gradient").

\section{Definition Of Lipschitz Constant $\mathcal{L}$}

A (positive) real number $\mathcal{L}$ is called a Lipschitz constant on a function $\mathrm{f}(\mathrm{x})$ in a region $\mathcal{R}$, if given any two points $\mathrm{x}_{1}$ and $\mathrm{x}_{2}$ in $\mathcal{R}$, the following condition holds:

$$
\left\|f\left(\mathbf{x}_{1}\right)-f\left(\mathbf{x}_{2}\right)\right\|<\mathcal{L}\left\|\mathbf{x}_{1}-\mathbf{x}_{2}\right\|
$$

where $\|$.$\| is a vector norm.$

If the constant $\mathcal{L}$ exists, a lipschitz condition is said to exist on the function $f(\mathbf{x})$ in the region $\mathcal{R}$. (See Figure 4.)

We also note that other schemes based on Lipschitz Constants have been used for accurate sampling of parametric surfaces by [VON HERZEN 87], [VON HERZEN 88] and [VON HERZEN 89].

\subsection{LG-surface Description}

We define an LG surface to be an implicit function $f(x, y, z)$ which has bounds on the net rate of change of the function and its directional derivative (that we call $L$ and $G$ ). Mathematically, these bounds are the Lipschitz constants as defined above; Lipschitz constants have other useful applications in applied mathematics and numerical analysis (see [GEAR 71] and [LIN AND SEGEL74])

\section{Definition of LG-surfaces}

Let $\mathrm{L}$ be the Lipschitz constant for the function $f(x)$ in a threedimensional region $\mathcal{R}$ and $\mathbf{G}$ be the Lipschitz constant for the corresponding function $g(t)$ in a closed interval $T=\left[t_{1}, t_{2}\right]$, ie.,

$$
\begin{aligned}
\left\|f\left(\mathbf{x}_{a}\right)-f\left(\mathbf{x}_{b}\right)\right\| & \leq L\left\|\mathbf{x}_{a}-\mathbf{x}_{b}\right\| \\
\left\|g\left(t_{a}\right)-g\left(t_{b}\right)\right\| & \leq G\left\|t_{a}-t_{b}\right\| .
\end{aligned}
$$

for any $x_{a}, x_{b} \in \mathcal{R}$ and any $t_{a}, t_{b} \in T$

An implicit surface $S$ represented by an implicit function $f(x)=0$ is an LG-implicit surface, if the Lipschitz constants $L$ and $\mathbf{G}$ as defined above exist and are computable.

\subsection{How To Compute L's and G's}

It can be seen from equation (3) that a Lipschitz constant is a measure of the maximum rate of change of a function in a region over which the function is defined. This can be seen by dividing equation 3 by $\left\|\mathbf{x}_{1}-\mathbf{x}_{2}\right\|$, taking the limit as $\mathbf{x}_{1} \rightarrow \mathbf{x}_{2}$ and using the definition of derivative.

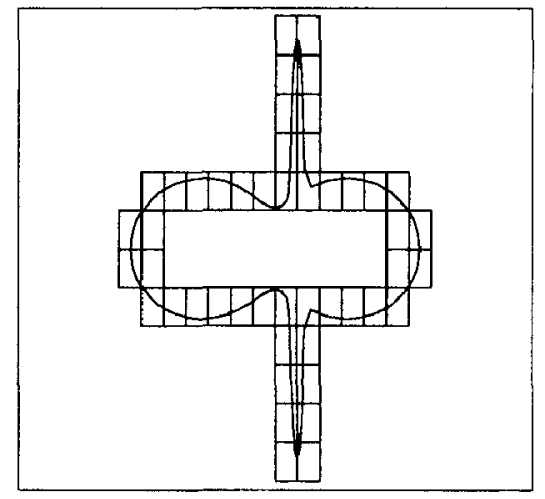

Figure 5: The straddling boxes of a surface as found by part A, Space Pruning step of the algorithm. The figure shows a two-dimensional slice through the three dimensional collection of boxes.

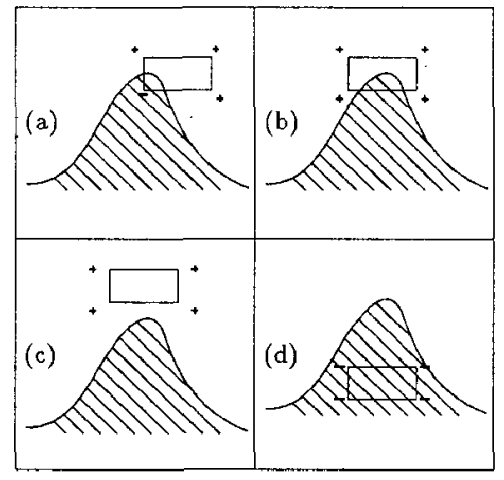

Figure 6: What is straddling? The box vertices straddle the surface if at least one of the vertices of the box is inside and at least one of the vertices is outside the surface (case (a)). Cases (b), (c) and (d) are all non-straddling.

\section{Computing L:}

Given a three dimensional rectangular region $\mathcal{R}$, we shall call the Lipschitz constant for $f(\mathbf{x})$ for $\mathcal{R}$ to be $\mathrm{L}$. $\mathrm{L}$ is equal to or greater than the maximum rate of change of $f(\mathbf{x})$ in $\mathcal{R}$. That is

$$
L \geq \max _{\mathcal{R}}|\nabla f(\mathbf{x})|
$$

\section{Computing G:}

Given a one dimensional closed interval $T=\left[t_{1}, t_{2}\right]$, we call the Lipschitz constant for $g(t)$ in $\mathcal{T}$ to be $\mathrm{G}$. T is equal to or greater than the maximum rate of change of $g(t)$ in $\mathcal{T}$. That is

$$
G \geq \max _{\mathcal{T}}\left|\frac{d g}{d t}\right|
$$

The algorithm works faster for smaller values of $L$ and $G$.

\section{Rendering LG-surfaces}

We render LG-surfaces by casting rays and obtaining intersections of the ray with the surface using two algorithms, $A$ and $B$.

Algorithm A) Space Pruning

Algorithm $A$ is an efficiency measure. Even if algorithm $A$ is not used, the ray intersection method in algorithm $B$ is guaranteed to work.

This algorithm prunes away large parts of space that are guaranteed not to contain any part of the LG-surface as in Figure 5. We obtain a. volume $\mathcal{V}$, composed of non-overlapping rectangular boxes, that contains the whole surface. The vertices of each of the boxes in $\mathcal{V}$ straddle the surface (figure 6). This initial pruning reduces the space in which we have to search for intersections of rays with the surface. 


\section{Algorithm B) Ray Intersection}

In algorithm $\mathbf{B}$, the volume $\mathcal{V}$ in space generated in the algorithm $\mathbf{A}$ is used to intersect a ray with the surface. The rectangular boxes composing $\nu$ are intersected with a ray in order of appearance along the ray. In any box, if one or more intersection exists, the nearest intersection is determined and we are done with this ray. The algorithm guarantees that if an intersection in the box exists, the intersection is found. Further, if more than one intersection exists, the nearest one is computed (nearest to the origin $t=0$ of the ray $\alpha t+\boldsymbol{\beta}$ ).

We now discuss each algorithm in more detail.

\subsection{Part A: Pruning Away Empty Regions Of Space}

The vertices of a rectangular box are said to straddle an implicit surface if at least one of the vertices lie inside the surface and at least one of the vertices lie outside. In Figure 6, part (a) shows a straddling box. We do not consider the box vertices in part (b) to be straddling even though the box itself contains a part of the surface. Boxes (c) and (d) are obviously non-straddling. The sign of $f(\mathrm{x})$, the inside-outside function for the surface tells if the point $\mathbf{x}$ is inside or outside the surface.

Algorithm A (Space Pruning) of the algorithm is shown in pseudo code in Figure 7 and in pictures in Figure 8. In this algorithm, we start with a bounding box that surrounds the surface of interest (Figure 8 , step 1). This bounding box is subdivided (Figure 8, step 2) to some level $n$ and sub-boxes guaranteed not to contain any portions of the surface are thrown away ( $\left(\right.$ Figure 8 , step 6 Box $\left.B_{1}\right)$. Only boxes that straddle the surface are kept (Figure 8, step 4 Box $B_{4}$ ). By keeping the boxes that only straddle the surface, each box is guaranteed to contain a part of the surface and we get a collection of boxes that lie close to the surface.

Given a sub-box B, how do we decide if it is to be accepted and not pruned away?

If $B$ straddles the surface, it certainly contains a part of the surface and it is kept (Figure 8, step 4 box $B_{4}$ ).

If $B$ does not straddle the surface, it still might contain a portion of the surface (Figure 8. step 4 box $B_{3}$ ). The L Lipschitz constant tells us if $B$ does not contain any part of the surface: Let $\mathbf{x}_{0}$ be the center of $\mathrm{B}$, and $d$ be half the length of the principal diagonal of $\mathrm{B}$. Since the maximum rate of change with respect to distance of $f(\mathbf{x})$ is $\mathrm{L}$ and the maximum distance of any point in $B$ from $x_{0}$ is $d$, the maximum change in the value of $f(\mathbf{x})$ in B from $f\left(\mathbf{x}_{0}\right)$ is $L d$. Hence if

$$
\left|f\left(\mathrm{x}_{0}\right)\right|>L d
$$

then $f(\mathbf{x})$ is guaranteed to stay the same sign that it has at $\mathbf{x}_{0}$ and never assume a value of zero in $B$ and hence $B$ can be thrown away (fig 7, steps 5 and 6 , box $B_{1}$ )

If $B$ does not satisfy condition in equation (8), it is subdivided into eight sub-boxes and each of the sub-boxes is checked again to check that it satisfies either the straddling condition or equation (8). In Figure 8 , step $7, B_{3}$ is subdivided and upon subdivision we find some sub-boxes that straddle the surface. For box $B_{2}$, none of the sub-boxes straddle the surface and the subdivision is stopped after condition (8) is satisfied

Figure 5 shows a surface and its straddling box forming $\nu$ as found by our algorithm.

This initial subdivision in algorithm $A$ is not an essential requirement for the algorithm. It is guaranteed to work even if the initial subdivision level $n=0$, i.e., no initial subdivision of the bounding box is performed. The level of initial subdivision does effect the performance of the ray-intersection.

\section{Subdivision Termination}

Is it guaranteed that the subdivision algorithm terminates?

If any of the vertices of a box lies exactly on the surface, condition (8) will never be satisfied. Even if we compute the smallest possible $L$, we can meet the condition $\left|f\left(\mathbf{x}_{0}\right)\right|=L d$ rather than the inequality. Hence, we have to stop subdivision at some level if it does not stop in the natural course of the algorithm. Since we compute the intersections numerically, stopping the intersection when the box size becomes smaller than the tolerance of the numerical method is a natural condition to use. Experimentally, in all the pictures we have computed

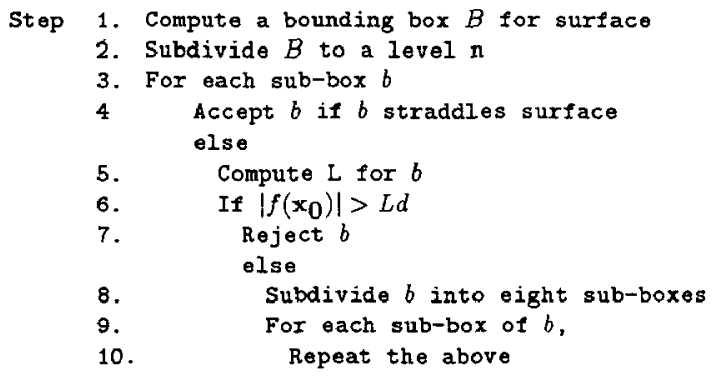
Figure 7: Algorithm A prunes away parts of space guaranteed not to contain any
portion of the surface. The algorithm is shown in pictures in Figure 8 . The steps portion of the surface. The algorithm is sh
in this figure and in Figure 8 correspond.

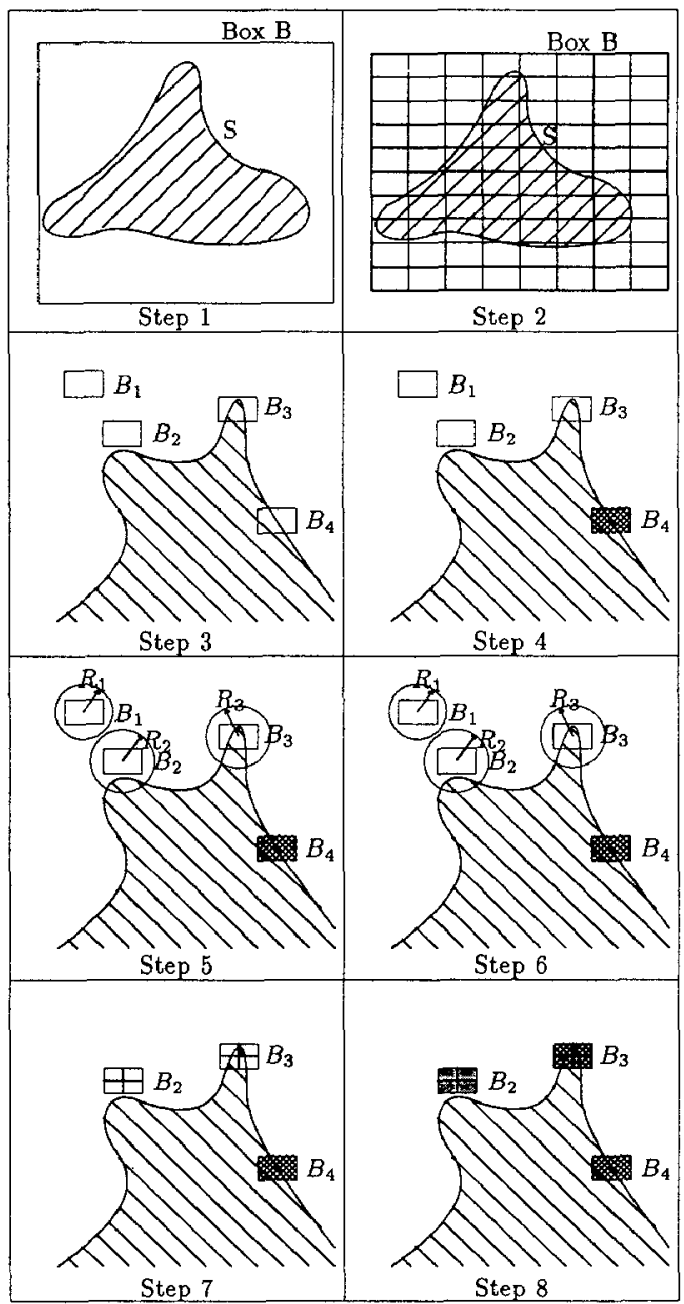

Figure 8: Algorithm A prunes away parts of space guraranteed not to contain any portion of an LG-surface. Cross hatched boxes are added to $\mathcal{V}$, lightly shaded boxes are thrown away. The pseudocode for the algorithm is given in Figure 7 . The steps in this figure and in Figure 7 correspond.

so far, such a terminating condition has never taken place. The straddling condition occurred before the box reached the numerical precision limit. 


\subsection{Part B: Intersection of a Ray with an LG- surface}

This part of the algorithm is designed to guarantee finding the intersection of a ray with an LG-surface nearest to the origin of the ray.

In algorithm $A$, we have formed a set of boxes that comprise a volume $\mathcal{V}$ and the vertices of each of the boxes in $\mathcal{V}$ straddle the surface. The volume $\mathcal{V}$ completely encloses the surface of interest. Hence all intersections lie in $\mathcal{V} . \mathcal{V}$ is composed of non-overlapping boxes. The ray intersection problem is now broken down into a simpler problem:

a) Find the box in $\nu$ nearest to the origin of the ray.

b) Compute any ray intersections in this box; if none exist, consider the next box in order.

The problem of finding the nearest box is described later in section 4. Here we consider the following problem.

\section{Problem Statement}

Intersection of a ray with the surface in a straddling box: Given a ray and a box $\mathrm{B}$ whose vertices straddle the surface, either ensure that there is no intersection of the ray with the surface in $B$ or find the nearest intersection.

\section{Preliminaries}

We wish to compute intersections along the ray $\boldsymbol{\alpha} t+\boldsymbol{\beta}$. Hence, we are interested in the behavior of $f(x)$ along the ray. As defined in equation $1, F(t)$ represents the behavior of $f(\mathbf{x})$ along the ray and

$$
g(t)=\frac{d F}{d t} .
$$

An intersection is given by values of $t$ such that

$$
F(t)=0
$$

The reliability of our ray intersection algorithm depends on our ability to determine if $g(t)$ becomes zero in an interval. The following two cases describe two important situations, one in which there is exactly one ray-surface intersection in an interval, and second in which there is none.

1. If $F\left(t_{1}\right)$ and $F\left(t_{2}\right)$ are of opposite signs at two points $t_{1}$ and $t_{2}$ along the ray, $t_{1}<t_{2}$, there is at least one intersection between $t_{1}$ and $t_{2}$. Further, if $g(t)=d F / d t$ does not become zero between $t_{1}$ and $t_{2}$, there is exactly one intersection between the ray and the surface in the interval from $t_{1}$ to $t_{2}$ (see below ${ }^{1}$ ).

2. If $F\left(t_{1}\right)$ and $F\left(t_{2}\right)$ have the same sign at $t_{1}$ and $t_{2}$ along the ray, $t_{1}<t_{2}$ and $g(t)=d f / d t$ does not become zero between $t_{1}$ and $t_{2}$, there is no intersection between the ray and the surface in the interval from $t_{1}$ to $t_{2}$. and

Given that $G$ is the Lipschitz constant for $g(t)$ in an interval $\left[t_{1}, t_{2}\right]$

$$
t_{m}=\left(t_{1}+t_{2}\right) / 2 \quad d=\left(t_{2}-t_{1}\right) / 2
$$

if

$$
\left|g\left(t_{m}\right)\right|>G d
$$

in $\left[t_{1}, t_{2}\right]$, then $g(t)$ nevers becomes zero in the interval. ( $G$ is equal or more than the maximum rate of change of $g(t)$ and $d$ is the maximum distance along the ray from $t_{m}$.) $G d$ is the maximum possible change in $g(t)$ from $g\left(t_{m}\right)$.

'If a continuous function $f(t)$ at tains two consecutive zero values at $t_{1}$ and $t_{2}$, by the mean value theorem, there exists at least one point between $t_{1}$ and $t_{2}$ where $d f / d t=0$.

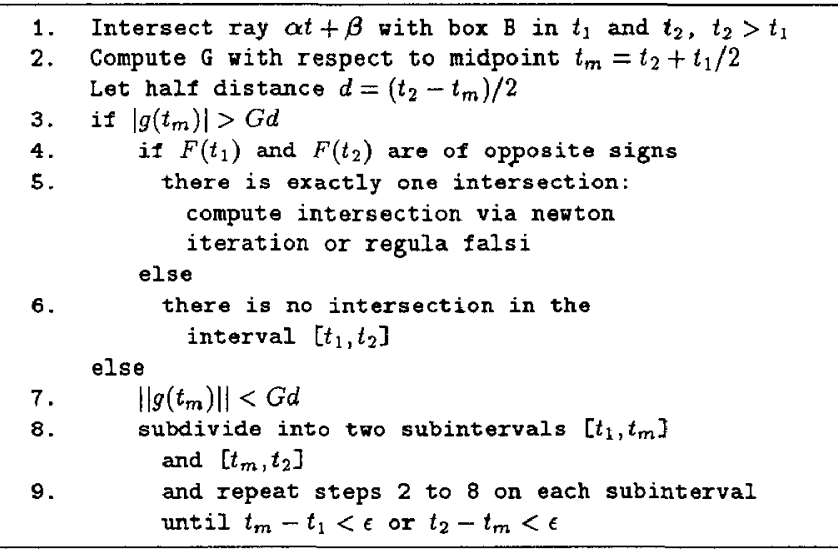

Figure 9: Algorithm to intersect a ray with an LG-surface. Given a box $B$, determine if there is no intersection in $B$ or else compute the nearest intersection. $\epsilon$ is the tolerance of the numerical method used to compute the intersection.

\section{B) Ray Intersection Algorithm}

The ray intersection algorithm is delineated in Figure 9.

We will use the above observations to design our ray intersection algorithm. We are looking for the interval $\left[t_{1}, t_{2}\right]$ nearest to the origin of the ray with exactly one intersection. Given a box $B$, we will make decisions about closest intersections of the surface $\mathrm{S}$ and the segment of the ray in the box.

Let the ray intersect box $\mathrm{B}$ in points $P_{1}$ and $P_{2}$ corresponding to the ray parameter values $t_{1}$ and $t_{2}$. Let $t_{m}$ be the midpoint of the segment between $t_{1}$ and $t_{2}$

$$
t_{m}=\frac{t_{2}+t_{1}}{2}, \quad d=\left(t_{2}-t_{1}\right) / 2
$$

\section{How to Find a Ray intersection}

1. If $F\left(t_{1}\right)$ and $F\left(t_{2}\right)$ are of opposite signs and $\left\|g\left(t_{m}\right)\right\|>G d$, there is exactly one intersection between $t_{1}$ and $t_{2}$. The intersection is computed numerically using a newton iteration and regula falsi method.

2. If $F\left(t_{1}\right)$ and $F\left(t_{2}\right)$ have the same sign and $\left\|g\left(t_{m}\right)\right\|>G d$, there is no intersection between $t_{1}$ and $t_{2}$. The next box $\mathrm{B}$ along the ray is considered. If there are no more boxes along the ray, the ray does not hit the surface.

If equation 9 is not satisfied in $\left[t_{1}, t_{2}\right]$, the interval is sudivided into two sub-intervals at $t_{m}$ and the two subintervals $\left[t_{1}, t_{m}\right]$ and $\left[t_{m}, t_{2}\right]$ tested in order.

\section{Interval Subdivision Termination}

The termination condition of the interval subdivision algorithm needs equation 9 to be satisfied. Since both $G$ and $d$ are positive, if $g(t)$ becomes exactly zero along the ray, this condition is never satisfied and the algorithm will not terminate naturally. This situation occurs at the silhouette edges of the surface ${ }^{2}$. At a point $P_{s}$ on the silhouette edge, the gradient of $f(x)$ is perpendicular to the ray and hence the directional derivative $g(t)$ is zero. Hence at $P_{s}$ condition (9) will never be satisfied.

To avoid an infinite subdivision, one has to stop when $\left|t_{2}-t_{1}\right|$ becomes less than the precision of the numerical method used to compute the intersections, typically of the order of $10^{-8}$. The surface will be smooth within the same error tolerance. The above considerations also imply that the algorithm spends more time near the silhouette edges. This is shown in Figure 12.

\subsection{Note about the Algorithm}

Note that the Space Pruning algorithm has to be done only once for each surface. The same volume $\mathcal{V}$ can be used repeatedly to make multiple pictures of that surface. Hence if we wish to make many

\footnotetext{
${ }^{2} \mathrm{~A}$ point $P_{s}$ on a surface $S: f(\mathbf{x})=0$ is said to be on a silhovette edge with respect to a view point $P_{v}$, if the ray originating from $P_{v}$ and passing through $P_{v}$ is tangent to $S$.
} 


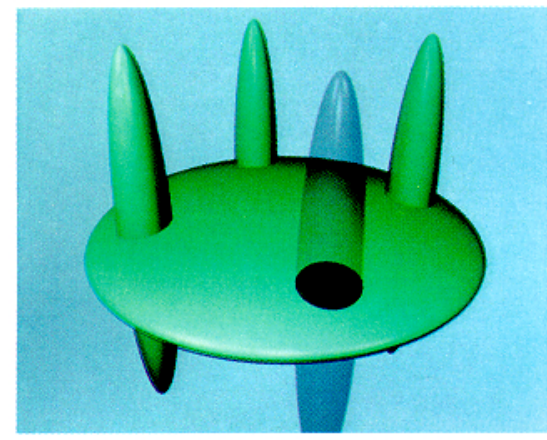

Figure 10: The proccdirc of creating the blended solid of Figure 11. The transparent ellipsoid is a negative function. All other ellipsoids are positive ellipsoids. Each ellipsoid has a different "blending factor".

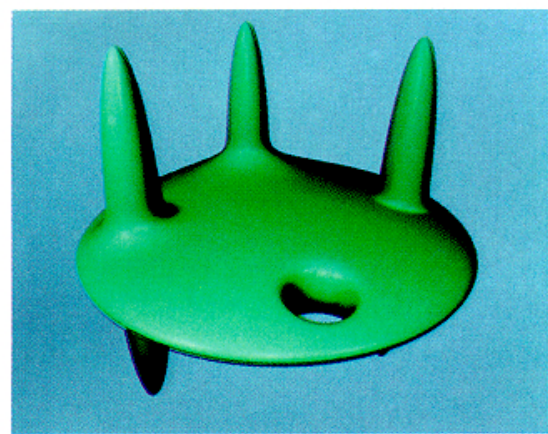

Figure 11: A blended solid made out of four positive ellipsoids and one negative solid. The whole solid is defined by one composite implicit function.

images of the same surface as say, in a movie, we should spend a little more extra computation in the Space Pruning algorithm by using a finer initial subdivision (since finer boxes save computation in the rayintersection algorithm).

\section{Implementation Details of the LG- Surface Ray Intersection Algorithm}

In this section we describe some details of our implementation of the algorithm and the kind of surfaces that we have considered so far for implementation. We are building a library of L's and G's for various functions that will be useful to implement more surfaces.

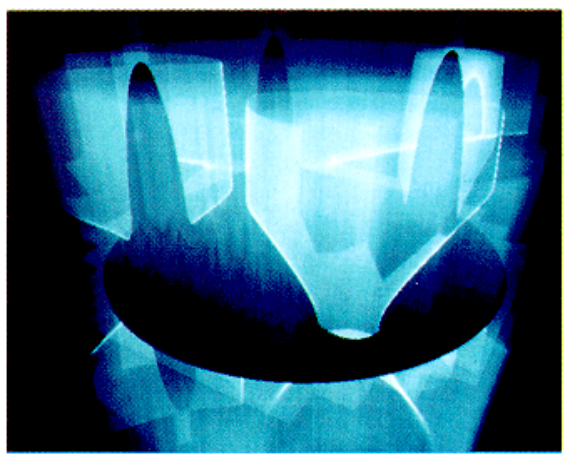

Figure 12: Pseudo color picture of Figure 11 showing that the ray-intersection algorithm spends more time at the silhouette edges. Brighter regions consumed more time.

\subsection{Implementing A New Surface}

To add a new surface to be rendered by our algorithm, the following functions have to be defined for the surface

double inside_outside $(x, y, z)$

double $x, y, z$;

inside_outside() returns the value of the implicit inside-outside function $f(\mathbf{x})$ of the surface. $f(\mathbf{x})$ is negative for points inside the surface and positive for points outside.

double lipschitz_constant (box_min, box_max)

double box_min [3], box max [3] :

lipschitz_constant() returns the value of a Lipschitz Constant for the surface in the box bounded by two vertices box_max and box_min Note that the value returned need not be the best estimate of the Lipschitz Constant. In fact, even a global Lipschitz Constant may be returned everytime. However, the algorithm is computationally more efficient for tighter estimates of Lipschitz Constants. The correctness of the algorithm remains unchanged irrespective of the tightness of the estimate of the Lipschitz constant.

double grad_lipschitz_constant(ray, t1, t2)

raytype ray

double t1, t2;

grad_lipschitz_constant() returns the value of a Lipschitz Constant $G$, the directional derivative of $f(x)$ along the ray, between the ray parameters $t 1$ and $t 2$. Again, we do not need the tightest value of $\mathrm{G}$, although tight bounds will save computation.

\subsection{Computing A Bounding Box}

To start the algorithm, a bounding box of the surface is required. How a bounding box is computed, of course, depends on the type of surface being considered. It should be, however, noted that the algorithm will work on any given bounding box. The algorithm will just render all the portions of the surface in the given box. In addition, a conservative estimate of the bounding box, too large a bounding box, penalizes mostly the first algorithm. Since a major portion of the bounding box that is guraranteed not to contain any part of the surface is pruned away in the first algorithm, the ray intersection in the second algorithm does not lose in computational efficiency much even with a large initial bounding box.

\subsection{Surfaces Considered So Far}

Admittedly, computing L's and G's for a general function $f(x)$ might require some mathematical prowess. We have computed L's and G's for some special surfaces to test our algorithm and have computed a series of images. As explained in the future work section, we are enlarging the library of available surfaces. This will be available in a forthcoming Caltech Technical Report.

The first example is an ellipsoid for which

$$
f(x, y, z)=a x^{2}+b y^{2}+c z^{2}-1,
$$

For a rectangular region $\mathcal{R}$ bounded by $\left(x_{\min }, y_{\min }, z_{\min }\right)$ and $\left(x_{\max }, y_{\max }, z_{\max }\right), L$ is given by,

$$
L_{\mathcal{R}}=2 \sqrt{a^{2}|x|_{\text {max }}^{2}+b^{2}|y|_{\text {max }}^{2}+c^{2}|z|_{\text {max }}^{2}}
$$

$G$ for an ellipsoid is independent of where it is computed along a ray $(x, y, z)=\alpha t+\beta$ and is given by

$$
G=2\left(a \alpha_{x}^{2}+b \alpha_{y}^{2}+c \alpha_{z}^{2}\right)
$$

To make more interesting surfaces, we have considered generalized algebraic surfaces as presented by Blinn in [BLINN 82]. We have extended them to non-spherical inside-outside functions.

\section{Generalized Algebraic Surfaces}

We briefly discuss the concept of Blinn's generalized algebraic surfaces.

Given a scalar function $f(x, y, z)$, he defines a new function,

$$
e(x, y, z)=B e^{A f(x, y, z)},
$$

where $A$ and $B$ are constants. 


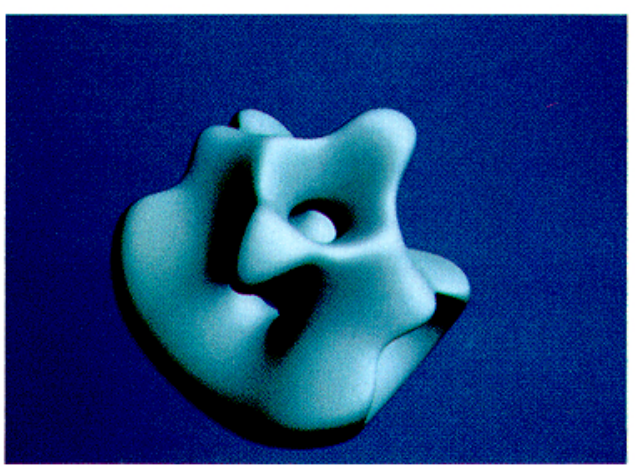

Figure 13: A surface perhaps reminiscent of biological or anatomical structures.

He then defines a sum function,

$$
P(x, y, z)=\sum_{i=1}^{n} B_{i} e^{A_{i} f_{i}(x, y, z)}
$$

The function $P(x, y, z)$ is a "blended" version of the individual functions $f_{i}(x, y, z)$. The exponentiation causes the blending to be gradual The parameters $A_{i}$ control the smoothness and parameters $B_{i}$ control the relative contribution of the participating functions $f_{i}$. Note that $B_{i}$ 's could be negative enabling one to "carve" out material from another solid. An example with positive and negative $B_{i}$ 's is shown in Figure 11 .

\section{L, G's for generalized algebraic surfaces}

We have computed L's and G's for blended surfaces comprised of ellipsoids, superquadrics and some other implicit surfaces. These results are given in appendices A.2.1 and A.2.2 and in [KALRA and BARR 89]. These results can be used to compute any of the pictures in this paper.

\subsection{Organizing Sub-boxes In An Octree}

The boxes generated in the Space Pruning algorithm and used in the ray intersection algorithm are arranged in an octree. Although octrees have been used by various people such as [GLASSNER 84], our octrees differ in some respects. Each of the node in our octree corresponds to a rectangular box. The children of a node are sub-boxes that straddle the surface.

Our octrees are not fully populated. Since the boxes that comprise the volume $\mathcal{V}$ generated in the first algorithm tend to be near the surface (because of the straddling condition), a large portion of the bounding box of the surface does not contain any sub-boxes in $\mathcal{V}$. Hence, although each of the nodes in our octree may potentially contain eight sub-nodes, most nodes will have fewer.

We also adjust the box of each node to tightly bound the boxes of its children nodes. Since we keep only straddling boxes ont of the eight possible boxes of a node, many nodes have fewer than eight children and the box for a node shrinks in a lot of cases. See Figure 14. In the figure the dotted boxes show the boxes before shrinking and the solid boxes after shrinking. This shrinking is propagated all the way to the root of the octree. During ray-intersection, the smaller boxes result in many more trivial rejects and we do not have to uselessly examine the children of many nodes.

The octree organization of boxes presents a convenient way to find the box nearest to the origin along a ray. If the ray does not intersect the bounding box of any node $N$, the sub-tree rooted at $N$ does not need to be considered. If the ray does hit the node box, the ray is intersected with each of its possible eight children, the boxes that are hit are arranged in a heap sorted by the near intersection of the ray with the box, and the boxes in the heap are tried in order.

\section{Results}

We have used the algorithm presented in this paper to compute a series of pictures shown in this paper. Figures 11 to 20 were all computed using our algorithm. Compare Figures 2 and 15. Our algorithm takes

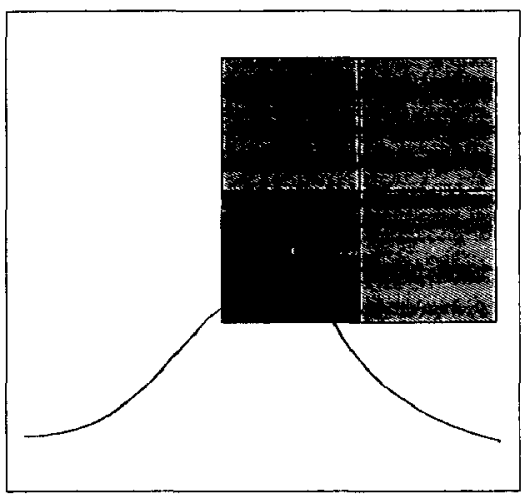

Figure 14: Shrinking of Parent Nodes. The bounding box of a parent is shrunk to the union of the boxes of its children nodes. The shrinking is propagated all the way to the root. The figure shows two levels of subdivision. The darkest shaded box represents the box to which the bounding boxes of both parent nodes are shrunk.

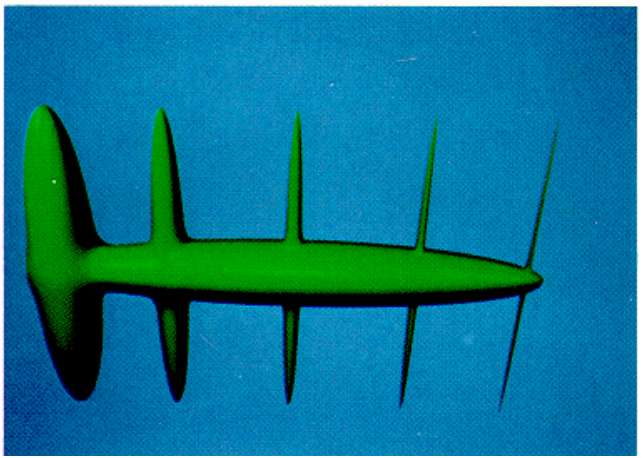

Figure 15: A row of spikes of decreasing thickness. The algorithm has no problem finding the smallest features.

care of the problem of missing fine features in Figure 2. The spikes in the picture change from thick to thin and the algorithm has no problem finding the finest of them without any fine tuning. The spikes in Figure 15 may be as fine as desired and the algorithm will find them. As advertized, we have found that the algorithm produces accurate intersections for all surfaces for which we can compute L's and G's.

We can use the algorithm presented in this paper even for functions that have not yet been analyzed. Since the L's and G's required need not be the smallest possible, we can use conservative global numbers and still make images of surfaces represented by these functions. We would have to spend extra computational effort because of large L's and G's. This approach, however, is an "indirect approach" and might

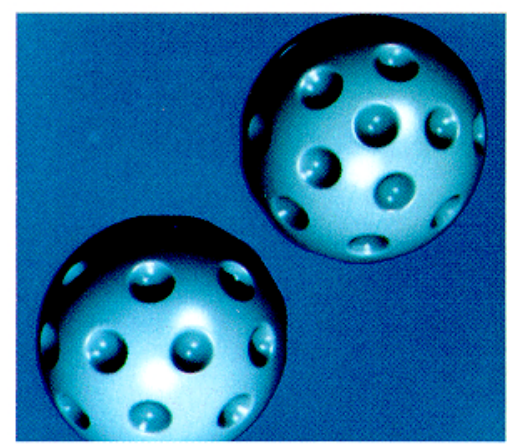

Figure 16: A golf ball composed of a positive sphere and smaller negative spheres. 


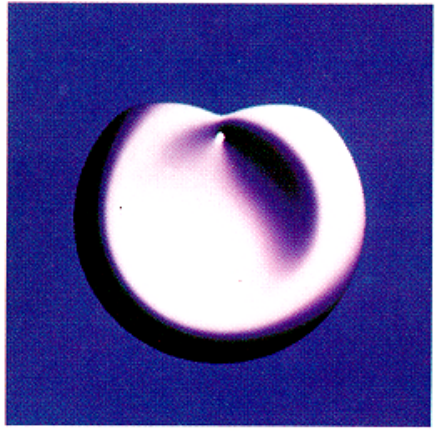

Figure 17: This implicit surface is generated by subtracting two spheres from a sphere. Note the thin bridge and the hole. Such fine features would be missed by most ad-hoc algorithms.

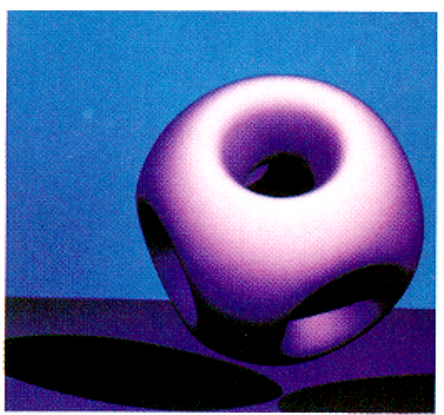

Figure 18: Another surface generated by combinations of positive and negative implicit functions.

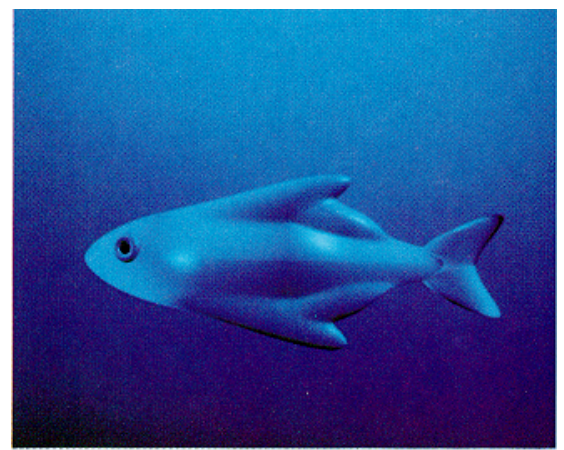

Figure 19: A fish generated by combinations of superquadratics and ellipsoids.

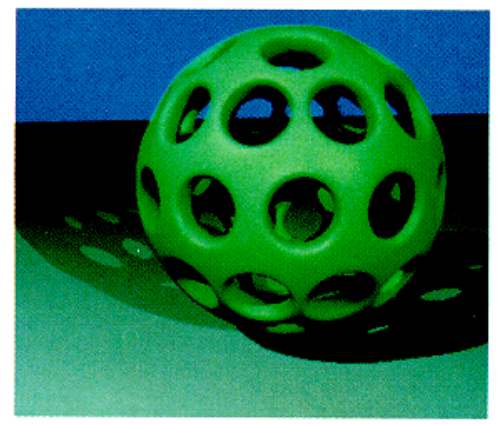

Figure 20: A wifle ball is created from a large solid sphere from which we subtract 25 ellipsoids and one smaller sphere.

require some fine tuning in the event that we choose too low estimates for L's and G's and thus obtain errors in the images.

\section{Conclusions}

In this paper we have presented a method to produce guaranteed intersections with a broad class of Implicit Surfaces. The most important feature of this algorithm is that it is a robust "direct" method. The method does not require any fine tuning or human choice of inter active parameters. The picture of a surface within the bounding box provided for the surface is guaranteed to be numerically correct. Further, if $\mathrm{L}$ and $\mathrm{G}$ 's can be computed locally for regions rather than globally, the algorithm adapts the computational effort required over different regions of the surface automatically.

Essentially, our algorithm is based on fundamental mathematical properties of functions and is correct by construction. With the algorithm presented in this paper, we have reduced the problem of ray tracing difficult implicit surfaces to the problem of finding L's and G's for the function representing the surface.

\section{Future Work}

Our algorithm presents an opportunity to use implicit surfaces as a useful tool for computer modeling. Our future work is directed in two directions.

First, we are working towards creating or identifying a collection of useful implicit functions and computing the Lipschitz constants $\mathrm{L}$ and $\mathrm{G}$, for these functions. We have already had some success in computing the desired Lipschitz constants for deformations, like twists, bends and tapers (See [BARR 84] for details of deformations)

Second, we are looking at applications of implicit surfaces where our algorithm can be used with advantage to render the surfaces. The picture in Figure 13 has inspired us to look at biological applications. We are considering modeling bones and other anatomical structures using blended surfaces as used in this paper. Another application is to use energy-based constraint methods ([TERZOPOULOS et al] and [PLATT and BARR]) to fit blended surfaces to digital data such as generated in tomography.

Another possible application is in adaptive anti-aliasing. Given a box, our algorithm can find if there is any surface in the box. Given that rays passing through the corners of a pixel miss the surface, an extension of this algorithm can be used to determine if there are any portions of the surface in the interior of the pixel and to possibly cause subdivision.

We have also been considering computational improvements to the algorithm. Computing a single number $\mathrm{L}$ for a rectangular box B actually gives us the radius of the circumscribing sphere $S$ of the box as shown in Figure 4. Since we use only the box, the region in the sphere $\mathrm{S}$ outside $\mathrm{B}$ is wasted. Using three Lipschitz constants $L_{1}, L_{2}$ and $L_{3}$, one for each of the three coordinate directions in place of a single $\mathrm{L}$ will make the algorithm more efficient. This is in some ways similar to methods in [VON HERZEN 89] and [VON HERZEN 88]. 


\section{Acknowledgements}

We would like to thank Steve Gabriel for his helpful comments and also to Harold Zatz. Thanks also are due to our sponsors for this work, Apple Computer, AT\&T, Hewlett Packard, IBM, and the National Science Foundation.

\section{A Computing $\mathrm{L}$ and $\mathrm{G}$}

\section{A.1 Lipschitz Constants For Sum Of Functions}

Given a set of functions $f_{1}(\mathbf{x}), f_{2}(\mathbf{x}), \ldots, f_{n}(\mathbf{x})$, with Lipschitz constants $L_{1}, L_{2}, \ldots, L_{n}$ respectively in a region $\mathcal{R}$, define the sum function $F_{0}$ as

$$
F_{s}=\sum_{i=1}^{n} f_{i}(x) \text {. }
$$

Then the Lipschitz constant $L_{s}$ of $F_{s}(\mathbf{x})$ in $\mathcal{R}$ satisfies the relation,

$$
L_{3} \leq \sum_{i=1}^{n} L_{i}
$$

\section{A.2 Computing $\mathrm{L}$ and $\mathrm{G}$ for generalized algebraic} surfaces

A.2.1 Case 1: Ellipsoidal Blending Functions

An ellipsoidal blending function is defined as

$$
f(x, y, z)=B e^{-A r(x, y, z)}
$$

where

$$
r(x, y, z)=a^{2} x^{2}+b^{2} y^{2}+c^{2} z^{2}
$$

We sketch out the derivation of computing $L$ and $G$ here. For details see the Caltech CS Technical Report [KALRA and BARR 89].

\section{Computing $L$ in a region $R$}

The $L$ lipschitz constant is computed by computing the gradient of $f(x, y, z)$ in equation 12 , taking its magnitude and maximizing it over a rectangular region.

For a rectangular region $\mathcal{R}$ bounded by $\left(x_{\min }, y_{\min }, z_{\min }\right)$ and $\left(z_{\max }, y_{\max }, z_{\max }\right), L$ is given by,

$$
L_{R}=2 A B R_{\max } e^{-A r_{\min }}
$$

where

$$
R=\sqrt{a^{4} x^{2}+b^{4} y^{2}+c^{4} z^{2}} .
$$

Note that $R_{\max }$ and $r_{\min }$ occur at the maximum and minimum distances respectively of any point in $\mathcal{R}$ from the origin. Note that, if the region $\mathcal{R}$ straddles any of the coordinate planes, $r$ does NOT take its minimum value at $\left(x_{\min }, y_{\min }, z_{\min }\right)$ which is one of the vertices of $\mathcal{R}$.

Computing $\mathrm{G}$ in an interval $\mathcal{T}=\left[t_{1}, t_{2}\right]$

The $G$ lipschitz constant is computed by substituting $(x, y, z)=\alpha t+\beta$ in equation 12 to obtain $F(t)$, differentiating to obtain $g(t)=d F / d t$ and maximizing $d g / d t$ over the interval $\left[t_{1}, t_{2}\right]$.

Define

where

$$
h(t)=-2 A B e^{-A r}\left(k_{1}-A\left(k_{1} t+k_{2}\right)^{2}\right)
$$

$$
\begin{aligned}
& k_{1}=a^{2} \alpha_{x}^{2}+b^{2} \alpha_{y}^{2}+c^{2} \alpha_{x}^{2} \\
& k_{2}=a^{2} \alpha_{x} \beta_{x}+b^{2} \alpha_{y} \beta_{y}+c^{2} \alpha_{z} \beta_{z}
\end{aligned}
$$

Thus

$$
G=\max _{t \in \mathcal{T}} h(t)
$$

But $h(t)$ can have a maximum value only at the endpoints $t_{1}$ or $t_{2}$, or else at a stationary point of $h(t)$,

$$
\begin{aligned}
& t_{3}=\frac{-\sqrt{3 k_{1} / 2 A}-k_{2}}{k_{1}} \\
& t_{4}=\frac{-k_{2}}{k_{1}} \\
& t_{5}=\frac{\sqrt{3 k_{1} / 2 A}-k_{2}}{k_{1}}
\end{aligned}
$$

See Figure 21.

Thus

$$
G=\max _{t_{i} \in \mathcal{T}} h\left(t_{i}\right)
$$

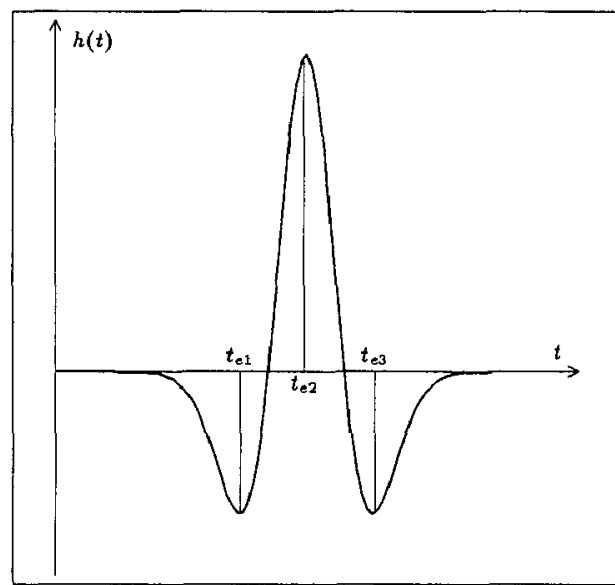

Figure 21: Plot of $h(t)=d g(t) / d t$ along a ray. To compute G, pick the maximum value of $h(t)$ in any interval $\left(t_{1}, t_{2}\right)$.

\section{A.2.2 Case 2: Superquadratic Blending Functions}

An superquadric blending function is defined as

$$
f(x, y, z)=B e^{-\operatorname{Ar}(x, y, z)}
$$

where

$$
r(x, y, z)=\left(x^{n}+y^{n}\right)^{\frac{m}{n}}+z^{m}
$$

The mathematics for $L$ and $G$ gets somewhat involved and we shall sketch out the derivation steps. Please see [KALRA and BARR 89] for full expressions and details.

\section{Method to Compute $L$ in a region $\mathcal{R}$}

Differentiate $f(x, y, z)$ with respect $\mathrm{x}, \mathrm{y}$ and $\mathrm{z}$ respectively and maximize the partial derivatives over a rectangular region $\mathcal{R}$ bounded by $\left(x_{\min }, y_{\min }, z_{\min }\right)$ and $\left(x_{\max }, y_{\max }, z_{\max }\right)$ to get $L_{1}, L_{2}$ and $L_{3}$ respectively.

$$
\begin{aligned}
& L_{1}=\max \left(m A B e^{-A r}\left[\left(x^{n}+y^{n}\right)^{\frac{m}{n}-1} x^{n-1}\right]\right) \\
& L_{2}=\max \left(m A B e^{-A r}\left[\left(x^{n}+y^{n}\right)^{\frac{m}{n}-1} y^{n-1}\right]\right) \\
& L_{3}=\max \left(m A B e^{-A r} z^{m-1}\right)
\end{aligned}
$$

We then use

$$
L=\sqrt{L_{1}^{2}+L_{2}^{2}+L_{3}^{2}}
$$

Method to Compute $\mathrm{G}$ in an Interval $\left[t_{1}, t_{2}\right]$

To compute G, substitute

$$
(x, y, z)=(\alpha t+\beta)
$$

in $f(x, y, z)$. Differentiate to get $g(t)$. Differentiate again and maximize over an interval $\left[t_{1}, t_{2}\right]$.

We found the use of a symbolic manipulation package such as [SMP] very useful in substituting for common sub-expressions etc. during the derivation of L's and G's.

Again, for more details see the Caltech CS Technical Report [KALRA and BARR 89].

\section{A.3 Lipschitz Constants For Deformations}

Let $h(x, y, z)$ be an inside/outside function. Let $\mathrm{D}(x, y, z)$ be a deformation function. The deformation function $D(x)$ maps a 3 -d point to another $3-d$ point. Examples are taper, twist, and bend [BARR 84].

The inside-outside function of the surface generated by deforming $h(x, y, z)$ by the deformation $\mathbf{D}$ is given by

$$
f(x, y, z)=h\left(\mathbf{D}^{-1}(\mathbf{x})\right)
$$


Computing $\mathrm{L}$ in a region $\mathcal{R}$

A Lipschitz constant $L$ for a region $V$ is computed as

$$
L \geq \max _{x \in V}\left\|\frac{\partial f}{\partial x_{i}}\right\| .
$$

where $\|\cdot\|$ represents a vector norm.

Computing $G$ in an interval $\left[t_{1}, t_{2}\right]$

Define

$$
g(t)=\frac{d}{d t} f(\alpha t+\beta) .
$$

for a ray $x=\alpha t+\theta$

Then $G$ for an interval $T$ along the ray is computed as

$$
G \geq \max _{t \in \mathcal{T}}\left|\frac{d g(t)}{d t}\right|
$$

The detailed derivation and expressions for $\mathrm{L}$ and $\mathrm{G}$ may be found in [KALRA and BARR 89].

\section{References}

[BARR 81] Alan H. Barr, Superquadrics and Angle-Preserving Transformations, IEEE Computer Graphics and Applications, Jan '81.

[BARR 84] Alan H. Barr, Global And Lacal Deformations of Solid Primitives, Computer Graphics, July '84.

[BLINN 82] James F. Blinn, A generalization of Algebraic Surface Drawing, ACM Transactions on Graphics, Vol. 1, No. 3, July 1982, pp 235-256.

[BLOOMENTHAL 85] Jules Blomenthal, Modeling the Mighty Maple, Computer Graphics, Vol 19, No. 3, July 1985.

[BLOOMENTHAL 87] Jules Blomenthal, Polygonization of Implicit Surfaces, Course Notes on "The Modeling of Natural Phenomena", Siggraph 1987.

[COLlins ANO AKRitas 76] Collins, G.E. and Akritas, A. G., Polynomial real root isolation using Descartes' rule of signs, Proc. 1976 ACM Symposium on Symbolic and Algebraic Computation.

[GEAR 71] Gear, C. William, Numerical Initial Value Problems in Ordinary Differential Equations, Prentice-Hall, Englewood Clifs, NJ, 1971.

[GLASSNER 84] Space Subdivision for Fast Ray Tracing, Andrew S. Glassner, IEEE Computer Graphics and Applications, October ' 84

[HANRAHAN 83] Pat Hanrahan, Ray Tracing Algebraic Surfaces, Computer Graphics. July' 83.

[KALRA and BARR 89] Devendra Kalra and Alan H. Barr, Guaranteed Intersections with Implicit surfaces, Caltech Computer Science Tech Report.

[LIN AND SEGEL74] Mathematics Applied To Deterministic Problems In The Natural Sciences, C.C. Lin and L. A. Segel, Macmillan Publishing Co., Inc., New York.

[LORENSEN AND CLINE 87] Marching Cubes: A high resolution 3d surface construction algorithm, William E. Lorensen and Harvey E. Cline, Computer Graphics, July 1987.

[MIDDLEDITCH et al 85] Alan E. Middleditch and Kenneth H. Sears, Blend Surfaces for Set theoretic volume Modeling Systems, Computer Graphics, Vol. 19, No. 3, July 1985, pp. 161-170.

[PLATT and BARR] John Platt and Alan Barr, Constraint Methods for Flexible Models, Computer Graphics, Aug 88.

[SMP] Steven Wolfram et al, SMP: A symbol manipulation Package, California Institute of Technology, 1981.

[TERZOPOUlos et al] Demetri Terzopoulos, John Platt, Al Barr, Kurt Fleischer, Elastically Deformable Models, Computer Graphics, July 87.

[USPENSKY 48] Uspensky, J. V., Theory of Equations, McGraw-Hill, 1948.

[VON HERZEN 84] Brian P. Von Herzen, Sampling Deformed, Intersecting Surfaces with Quadtrees, Master's Thesis, Caltech, 1984.

[VON HERZEN 88] Brian Von Herzen, Applications of Surface Networks to Sampling Problems in Computer Graphics, Caltech $\mathrm{Ph} \mathrm{D}$ Thessis.
[VON HERZEN 89] Brian Von Herzen, Alan H, Barr, Harold R. Zatz, Col. lision Determination for Parametric Surfaces, Caltech CS Technical Report.

[WYVILL 86] Space Division for Ray Tracing in CSG, Geoff Wyvill, Tosiyasu L. Kunii and Yasuto Shirai, IEEE Computer Graphics and Applications, April '86.

IWYVILL 87[1] Solid Texturing of Soft Objects, Geoff Wyvill, Brian Wyvill, Craig Pheeters, IEEE Computer Graphics and Applications, December ' 87 .

[WYVILL 87[2]] Animating Soft Objects, Geoff Wyuil, Craig Pheeters, Brian Wyvill, The Visual Computer, (1986)2. 